\title{
Radiation effect on unsteady MHD flow through porous medium past an oscillating inclined plate with variable temperature and mass diffusion in the presence of Hall current
}

\author{
U. S. Rajput and Gaurav Kumar * \\ Department of Mathematics and Astronomy, University of Lucknow, U.P, India. \\ * Corresponding author: rajputgauravlko@gmail.com
}

\section{Article history}

Received 5 Jun 2016

Accepted 30 Jun 2016

\section{Graphical abstract}

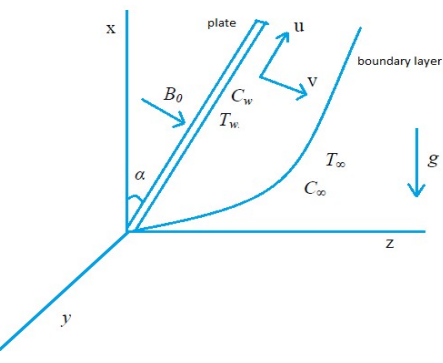

\begin{abstract}
In the present paper, we study the effect of radiation on unsteady flow of a viscous, incompressible and electrically conducting fluid past an oscillating inclined plate through a porous medium with variable wall temperature and mass diffusion in the presence of transversely applied uniform magnetic field. The plate temperature and the concentration level near the plate increase linearly with time. The governing equations involved in the present analysis are solved by the Laplacetransform technique. The results obtained are discussed with the help of graphs drawn for different parameters. The numerical values obtained for skin-friction and Nusselt number have been tabulated. The results are found to be in good agreement and the data obtained is in concurrence with the actual flow phenomenon.
\end{abstract}

Keywords: MHD flow, oscillating inclined plate, variable temperature, mass diffusion, Hall current.

\section{INTRODUCTION}

The influence of magnetic field on viscous incompressible flow of electrically conducting, radiative reactive fluid through porous medium associated with heat and mass transfer plays important role in different areas of science and technology. Radiation effect on MHD flow is also significant in many cases. Some such problems already studied are mentioned here. Radiation effect on combined convection over a vertical flat plate embedded in a porous medium of variable porosity was analyzed by $\mathrm{Pal}$ and Mondal [1]. They solved flow model by using finite difference method and observed that if radiation parameter is increased then the momentum and thermal boundary layer thickness increases. Manivannan et al. [2] have considered radiation and chemical reaction effects on isothermal vertical oscillating plate with variable mass diffusion. They solved dimensionless governing equations by using the Laplace transform method and found that the velocity and temperature of the fluid increases with decrease in radiation parameter. Radiative MHD flow over a non-isothermal stretching sheet in a porous medium was analyzed by Vyas and Srivastava [3]. They have used the finite difference scheme to solve the equations. They discussed that the temperature gradient increases with increase in the radiation parameter. Rajput and Kumar [4] have considered radiation effect on MHD flow through porous media past an impulsively started vertical plate with variable heat and mass transfer. They have used Laplace transform technique to solve governing equations, and found that the velocity of fluid near the vertical plate decreases with increase in radiation parameter. Reddy et al. [8] have worked on analytical study of MHD free convective, dissipative boundary layer flow past a porous vertical surface in the presence of thermal radiation, chemical reaction and constant suction. They have used regular perturbation technique to solve the governing equations. They found that velocity and temperature of the fluid near the plate diminishes with increase in radiation parameter. Diffusion-thermo and radiation effects on MHD free convection flow of chemically reacting fluid past an oscillating plate embedded in porous medium was studied by Swetha et al [9]. They solved flow model by using the Laplace transform technique, and discussed that the velocity curve rises in the case of heating plate and falls in the case of cooling plate with the increase in radiation. Also they observed that the skin friction always decreases for cooling plate and increases for heating plate with the increase in radiation. Balla and Naikoti [10] have investigated radiation effect on unsteady MHD convective heat and mass transfer past a vertical plate with chemical reaction and viscous dissipation. They have used finite element method based on Galerkin weighted residual approach to solve the governing coupled nonlinear boundary layer partial differential equations. They noticed that increase in radiation parameter reduces the velocity, temperature and concentration of the fluid near the plate. Ismail et al. [6] have considered MHD double diffusion flow by free convection past an infinite inclined plate with ramped wall temperature in a porous medium. They have used Laplace transform method to solve non-linear governing equations, and observed that the fluid velocity increases with decreasing radiation parameter. Some papers related with Hall effect are also mentioned here. Purkayastha and Choudhury [5] have investigated Hall current and thermal radiation effect on MHD convective flow of an elastico-viscous fluid in a rotating porous channel. They have used perturbation technique to solve non-linear governing equations, and observed that if radiation parameter is increased then the velocity of fluid increases. Hall effect on unsteady MHD free convection flow over a stretching sheet with variable viscosity and viscous dissipation was studied by Srinivas and Naikoti [7]. They solved flow model by 
the implicit finite difference method. In this paper, we are considering radiation effect on unsteady MHD flow through porous medium past an oscillating inclined plate with variable temperature and mass diffusion in the presence of Hall current. The results are shown with the help of graphs and table.

\section{MATHEMATICAL ANALYSIS}

Geometric model of the problem is shown in Figure-1

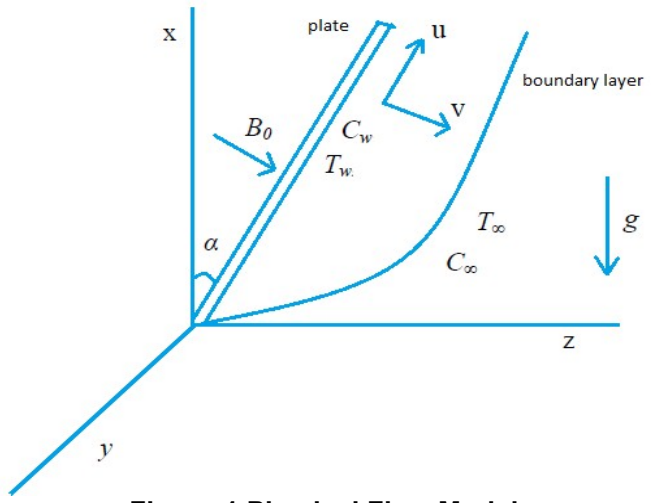

Figure-1 Physical Flow Model

The $\mathrm{x}$ axis is taken along the vertical plane and $\mathrm{z}$ axis is normal to it. Thus the $\mathrm{z}$ axis lies in the horizontal plane. The plate is inclined at angle $\alpha$ from vertical. The magnetic field $B_{0}$ of uniform strength is applied perpendicular to the flow. During the motion, the direction of the magnetic field changes along with the plate in such a way that it always remains perpendicular to it. This means, the direction of magnetic field is tied with the plate. Initially it has been considered that the plate as well as the fluid is at the same temperature $T_{\infty}$. The species concentration in the fluid is taken as $C_{\infty}$. At time $\mathrm{t}>0$, the plate starts oscillating in its own plane with frequency $\omega$, and temperature of the plate is raised to $T_{w}$. The concentration $C$ near the plate is raised linearly with respect to time. The flow modal is as under:

$$
\begin{aligned}
& \frac{\partial u}{\partial t}= v \frac{\partial^{2} u}{\partial z^{2}}+g \beta \operatorname{Cos} \alpha\left(T-T_{\infty}\right)+ \\
& g \beta^{*} \operatorname{Cos} \alpha\left(C-C_{\infty}\right)-\frac{\sigma B_{0}^{2}(u+m v)}{\rho\left(1+m^{2}\right)}-\frac{v u}{K}, \\
& \frac{\partial v}{\partial t}= v \frac{\partial^{2} v}{\partial z^{2}}+\frac{\sigma B_{0}^{2}(m u-v)}{\rho\left(1+m^{2}\right)}-\frac{v v}{K} \\
& \frac{\partial C}{\partial t}=D \frac{\partial^{2} C}{\partial z^{2}}, \\
& \rho C_{p} \frac{\partial T}{\partial t}=k \frac{\partial^{2} T}{\partial z^{2}}-\frac{\partial q_{r}}{\partial z} .
\end{aligned}
$$

The boundary conditions for the flow are as under:

$$
\begin{aligned}
& t \leq 0: u=0, v=0, T=T_{\infty}, C=C_{\infty} \text {, for every } z \text {, } \\
& \mathrm{t}>0: \mathrm{u}=\mathrm{u}_{0} \operatorname{Cos} \omega t, \mathrm{v}=0 \text {, } \\
& \mathrm{T}=\mathrm{T}_{\infty}+\left(\mathrm{T}_{\mathrm{w}}-\mathrm{T}_{\infty}\right) \frac{\mathrm{u}_{0}^{2} \mathrm{t}}{\mathrm{v}}, \quad \text { at } \mathrm{z}=0, \\
& \mathrm{C}=\mathrm{C}_{\infty}+\left(\mathrm{C}_{\mathrm{w}}-\mathrm{C}_{\infty}\right) \frac{\mathrm{u}_{0}^{2} \mathrm{t}}{\mathrm{v}}, \\
& u \rightarrow 0, v \rightarrow 0, T \rightarrow T_{\infty}, C \rightarrow C_{\infty} \text { as } z \rightarrow \infty \text {. }
\end{aligned}
$$

Here $\mathrm{u}$ is the primary velocity, $v$ - the secondary velocity, g-the acceleration due to gravity, $\beta$-volumetric coefficient of thermal expansion, t-time, $m\left(=\omega_{e} \tau_{e}\right)$-the Hall current parameter with $\omega_{e^{-}}$ cyclotron frequency of electrons and $\tau_{e}$-electron collision time, $T$ temperature of the fluid, $\beta^{*}$ - volumetric coefficient of concentration expansion, $C$ - species concentration in the fluid, $\nu$ - the kinematic viscosity, $\rho$ - the density, $C_{p}$ - the specific heat at constant pressure, $k$ thermal conductivity of the fluid, $D$-the mass diffusion coefficient, $K$ the permeability parameter, $T_{w}$ - temperature of the plate at $z=0, C_{w}$ species concentration at the plate $z=0, B_{0^{-}}$the uniform magnetic field, $\sigma$ - electrical conductivity.

The local radiant for the case of an optically thin gray gas is expressed by

$$
\frac{\partial q_{r}}{\partial z}=-4 a^{*} \sigma\left(T_{\infty}^{4}-T^{4}\right)
$$

where $a^{*}$ is absorption constant. The temperature difference within the flow is sufficiently small therefore, $T^{4}$ can be expressed as the linear function of temperature. This is accomplished by expanding $T^{4}$ in a Taylor series about $T_{\infty}$ and neglecting higher-order terms

$T^{4} \cong 4 T_{\infty}^{3} T-3 T_{\infty}^{4}$

Using equations (6) and (7), equation (4) becomes

$\rho C_{p} \frac{\partial T}{\partial t}=k \frac{\partial^{2} T}{\partial z^{2}}-16 a^{*} \sigma T_{\infty}^{3}\left(T-T_{\infty}\right)$

The following non-dimensional quantities are introduced to transform equations (1), (2), (3) and (8) into dimensionless form:

$$
\begin{gathered}
\bar{z}=\frac{z u_{0}}{v}, \bar{u}=\frac{u}{u_{0}}, \bar{v}=\frac{v}{u_{0}}, \theta=\frac{\left(T-T_{\infty}\right)}{\left(T_{w}-T_{\infty}\right)}, \\
S_{c}=\frac{v}{D}, \mu=\rho v, P_{r}=\frac{\mu c_{p}}{k}, M=\frac{\sigma B_{0}^{2} v}{\rho u_{0}^{2}} \\
R=\frac{16 a^{*} \sigma v^{2} T_{\infty}^{3}}{k u_{0}^{2}}, \bar{\omega}=\frac{\omega v}{u_{0}^{2}}, \bar{K}=\frac{u_{0}}{v^{2}} K \\
G_{m}=\frac{g \beta^{*} v\left(C_{w}-C_{\infty}\right)}{u_{0}{ }^{3}}, \bar{C}=\frac{\left(C-C_{\infty}\right)}{\left(C_{w}-C_{\infty}\right)} \\
G_{r}=\frac{g \beta v\left(T_{w}-T_{\infty}\right)}{u_{0}{ }^{3}}, \bar{t}=\frac{t u_{0}^{2}}{v} .
\end{gathered}
$$

Here $\bar{u}$ is the dimensionless primary velocity, $\bar{v}$-the secondary velocity, $\bar{t}$-dimensionless time, $\theta$-the dimensionless temperature, $\bar{C}$ -the dimensionless concentration, $G_{r}$-thermal Grashof number, $G_{m^{-}}$ mass Grashof number, $\mu$-the coefficient of viscosity, $\bar{K}$-the dimensionless permeability parameter, $P_{r}$-the Prandtl number, $S_{c}$-the Schmidt number, R-radiation parameter, $M$ - the magnetic parameter.

Thus the model becomes

$$
\begin{aligned}
\frac{\partial \bar{u}}{\partial \bar{t}}= & \frac{\partial^{2} \bar{u}}{\partial \bar{z}^{2}}+G_{r} \operatorname{Cos} \alpha \theta+G_{m} \operatorname{Cos} \alpha \bar{C}- \\
& \frac{M(\bar{u}+m \bar{v})}{\left(1+m^{2}\right)}-\frac{1}{\bar{K}} \bar{u},
\end{aligned}
$$




$$
\begin{aligned}
\frac{\partial \bar{v}}{\partial \bar{t}} & =\frac{\partial^{2} \bar{u}}{\partial \bar{z}^{2}}+\frac{M(m \bar{u}-\bar{v})}{\left(1+m^{2}\right)}-\frac{1}{\bar{K}} \bar{v}, \\
\frac{\partial \bar{C}}{\partial \bar{t}} & =\frac{1}{S_{c}} \frac{\partial^{2} \bar{C}}{\partial \bar{z}^{2}} \\
\frac{\partial \theta}{\partial \bar{t}} & =\frac{1}{P_{r}} \frac{\partial^{2} \theta}{\partial \bar{z}^{2}}-\frac{R \theta}{P_{r}} .
\end{aligned}
$$

The boundary conditions (5) become:

$\bar{t} \leq 0: \bar{u}=0, \bar{v}=0, \theta=0, \bar{C}=0$, for every $\bar{z}$, $\bar{t}>0: \bar{u}=\operatorname{Cos} \bar{\omega} \bar{t}, \bar{v}=0, \theta=\bar{t}, \bar{C}=\bar{t}$, at $\bar{z}=0$, $\bar{u} \rightarrow 0, \bar{v} \rightarrow 0, \theta \rightarrow 0, \bar{C} \rightarrow 0$,as $\bar{z} \rightarrow \infty$.

Dropping bars in the above equations, we get

$$
\begin{aligned}
\frac{\partial u}{\partial t}= & \frac{\partial^{2} u}{\partial z^{2}}+G_{r} \operatorname{Cos} \alpha \theta+G_{m} \operatorname{Cos} \alpha C- \\
& \frac{M(u+m v)}{\left(1+m^{2}\right)}-\frac{1}{K} u, \\
\frac{\partial v}{\partial t}= & \frac{\partial^{2} v}{\partial z^{2}}+\frac{M(m u-v)}{\left(1+m^{2}\right)}-\frac{1}{K} v, \\
\frac{\partial C}{\partial t}= & \frac{1}{S_{c}} \frac{\partial^{2} C}{\partial z^{2}}, \\
\frac{\partial \theta}{\partial t}= & \frac{1}{P_{r}} \frac{\partial^{2} \theta}{\partial z^{2}}-\frac{R \theta}{P_{r}},
\end{aligned}
$$

The corresponding boundary conditions are:

$\left.\begin{array}{l}t \leq 0: u=0, v=0, \theta=0, C=0, \text { for every z, } \\ t>0: u=\operatorname{Cos} \omega t, v=0, \theta=t, C=t, \text { at } \mathrm{z}=0, \\ u \rightarrow 0, v \rightarrow 0, \theta \rightarrow 0, C \rightarrow 0, \text { as } z \rightarrow \infty\end{array}\right\}$

Combining equations (15) and (16), the model becomes

$$
\begin{aligned}
& \frac{\partial q}{\partial t}=\frac{\partial^{2} q}{\partial z^{2}}+G_{r} \operatorname{Cos} \alpha \theta+G_{m} \operatorname{Cos} \alpha C-q a, \\
& \frac{\partial C}{\partial t}=\frac{1}{S_{c}} \frac{\partial^{2} C}{\partial z^{2}}, \\
& \frac{\partial \theta}{\partial t}=\frac{1}{P_{r}} \frac{\partial^{2} \theta}{\partial z^{2}}-\frac{R \theta}{P_{r}},
\end{aligned}
$$

Finally, the boundary conditions become:

$$
\begin{aligned}
& t \leq 0: q=0, \theta=0, C=0, \text { for every z, } \\
& t>0: q=\operatorname{Cos} \omega t, \theta=t, C=t, \text { at } \mathrm{z}=0, \\
& q \rightarrow 0, \theta \rightarrow 0, C \rightarrow 0, \text { as } z \rightarrow \infty
\end{aligned}
$$

Here $q=u+i v, a=\frac{M(1-i m)}{1+m^{2}}+\frac{1}{\mathrm{~K}}$.

The dimensionless governing equations (20) to (22), subject to the boundary conditions (23), are solved by the usual Laplace - transform technique.

The solution obtained is as under:

$$
\begin{aligned}
q= & \frac{1}{2} e^{-\sqrt{a} z} A_{33}+\frac{G_{r} \operatorname{Cos} \alpha}{4(a-R)^{2}}\left[2 e^{-\sqrt{a} z}\left(A_{1}+P_{r} A_{2}\right)\right. \\
& +2 t A_{2} e^{-\sqrt{a} z}(a-R)+z A_{3} e^{-\sqrt{a} z}\left(\sqrt{a}-\frac{R}{\sqrt{a}}\right) \\
& \left.+2 A_{12} A_{4}\left(1-P_{r}\right)\right]+\frac{G_{m} \operatorname{Cos} \alpha}{4 a^{2}}\left[e ^ { - \sqrt { a } z } \left(2 A_{1}+\right.\right. \\
& \left.2 \sqrt{a} A_{3}\right)+2 e^{-\sqrt{a} z} A_{2}\left(S_{c}+a t\right)+2 A_{13} A_{5}(1- \\
& \left.\left.S_{c}\right)\right]-\frac{P_{r} G_{r} \operatorname{Cos} \alpha}{2 \sqrt{\pi}(a-R)^{2} A_{11}}\left[A_{16} A_{6} \sqrt{\pi} z(a t-1\right. \\
& \left.-R t+P_{r}\right)+A_{14} A_{7} \sqrt{\pi} z\left(1-P_{r}\right)+\frac{1}{2} \sqrt{\frac{P_{r}}{R}} A_{16} A_{8} \\
& \left.A_{11} \sqrt{\pi} z(a-R)\right]-\frac{G_{m} \operatorname{Cos} \alpha}{2 a^{2} \sqrt{\pi}}\left[2 a z \sqrt{S_{c}} e^{-\frac{z^{2} S_{c}}{4 t}} \sqrt{t}\right. \\
& +A_{15} \sqrt{\pi}\left(a z^{2} S_{c}+2 a t+2 S_{c}-2\right)+A_{13} \sqrt{\pi}\left(A_{9}\right. \\
& \left.\left.+A_{10} S_{c}\right)\right], \\
C= & \left.t\left(1+\frac{z^{2} S_{c}}{2 t}\right) e r f c\left[\frac{\sqrt{S_{c}}}{2 \sqrt{t}}\right]-\frac{z \sqrt{S_{c}}}{\sqrt{\pi \sqrt{t}}} e^{-\frac{z^{2}}{4 t}} S_{c}\right\}, \\
\theta= & \frac{e^{-\sqrt{R} z}}{4 \sqrt{R}}\left\{e r f c\left[\frac{-2 \sqrt{R} t+z P_{r}}{\sqrt{P_{r} t}}\right](2 \sqrt{R} t-z R)+\right. \\
& \left.e^{2 \sqrt{R} z} e r f c\left[\frac{2 \sqrt{R} t+z P_{r}}{\sqrt{P_{r} t}}\right](2 \sqrt{R} t+z R)\right\} .
\end{aligned}
$$

The expressions for the constants involved in the above equations are given in the appendix.

\section{SKIN FRICTION}

The dimensionless skin friction at the plate $\mathrm{z}=0$ is obtained by

$\left(\frac{d q}{d z}\right)_{z=0}=\tau_{x}+i \tau_{y}$

Separating real and imaginary parts in $\left(\frac{d q}{d z}\right)_{z=0}$, the dimensionless skin - friction components $\tau_{x}=\left(\frac{d u}{d z}\right)_{z=0}$ and $\tau_{y}=\left(\frac{d v}{d z}\right)_{z=0}$ can be computed.

\section{NUSSELT NUMBER}

The dimensionless Nusselt number at plate $\mathrm{z}=0$ is given by 


$$
\begin{array}{r}
N u=\left(\frac{\partial \theta}{\partial z}\right)_{z=0}=\operatorname{erfc}\left[\frac{\sqrt{R} t}{\sqrt{t P_{r}}}\right]\left(\sqrt{R} t-\frac{\sqrt{R}}{2} t+\frac{P_{r}}{4 \sqrt{R}}\right) \\
-\operatorname{erfc}\left[-\frac{\sqrt{R} t}{\sqrt{t P_{r}}}\right]\left(\frac{\sqrt{R}}{2} t+\frac{P_{r}}{4 \sqrt{R}}\right)-\frac{e^{-\frac{R t}{P_{r}}} \sqrt{t P_{r}}}{\sqrt{\pi}}
\end{array}
$$

\section{RESULT AND DISCUSSION}

The velocity profiles for different parameters like thermal Grashof number $(G r)$, magnetic field parameter $(M)$, Hall parameter $(m)$, radiation parameter $(R)$, permeability parameter $(K)$, Prandtl number $(P r)$ and time $(t)$ are shown in figures 1.1 to 2.11 . Temperature profiles for different values of $P r, R$ and time are shown in figures 3.1 to 3.3. Due to gravity component $g \operatorname{Cos} \alpha$, the fluid flows with higher velocity when plate is vertical as compared to flow when plate is horizontal. It is observed from figures 1.1 and 2.1 that the primary and secondary velocities of fluid decrease when the angle of inclination $(\alpha)$ is increased. It is observed from figure 1.2 and 2.2, when the mass Grashof number is increased then the velocities are increased. From figures 1.3 and 2.3 it is deduced that velocities increase with thermal Grashof number $(\mathrm{Gr})$. Also, if Hall current parameter $(\mathrm{m})$ is increased then $\mathrm{u}$ increases, while $v$ gets decreased (figures 1.4 and 2.4). The influence of magnetic field on flow is observed from figures 1.5 and 2.5. It is seen that the effect of increasing values of the parameter $(M)$ results in decreasing $u$ and increasing $v$. It is in agreement since the magnetic field establishes a force which acts against the main flow resulting in slowing down the velocity of fluid. It is deduced that when radiation parameter $R$ is increased then the velocities are increased (figures 1.6 and 2.6). This implies that thermal radiation tends to accelerate primary and secondary fluid velocities throughout the boundary layer region near the plate. From figures 1.7 and 2.7, it is deduced that velocities increase with permeability parameter $(K)$. This result is due to the fact that increases in the value of $(K)$ results in reducing the drag force, and hence increasing the fluid velocity. If phase angle $(\omega t)$ is increased then velocities of fluid are decreased (figures 1.8 and 2.8). Further, it is observed that velocities decrease when Prandtl number and Schmidt number are increased (figures 1.9, $2.9,1.10$ and 2.10). Physically, the increase of $S c$ means decrease of molecular diffusivity $(D)$. This shows that the increase of $S c$ means decrease of molecular diffusivity $(D)$. That is the process of diffusion will decrease. Further, from figures 1.11 and 2.11 , it is observed that velocities increase with time. It is observed that temperature decreases when Prandtl number and radiation parameter are increased (figures 3.1 and 3.2). However, from figure 3.3 it is observed that temperature increases with time.

Skin friction is given in table1. The value of $\tau_{x}$ increases with the increase in $G r, G m, m, R, \omega t$ and $K$. The value of $\tau_{x}$ decreases with $\alpha$, $M, P r, S c$ and $t$. Similar effect is observed with $\tau_{y}$, except $M, m$ and $\omega t$, in which case $\tau_{y}$ increases with $M$, and decreases with $m$ and $\omega t$.

Nusselt number is given in table2. The value of $N u$ decreases with increase in $\operatorname{Pr}, R$ and $t$.

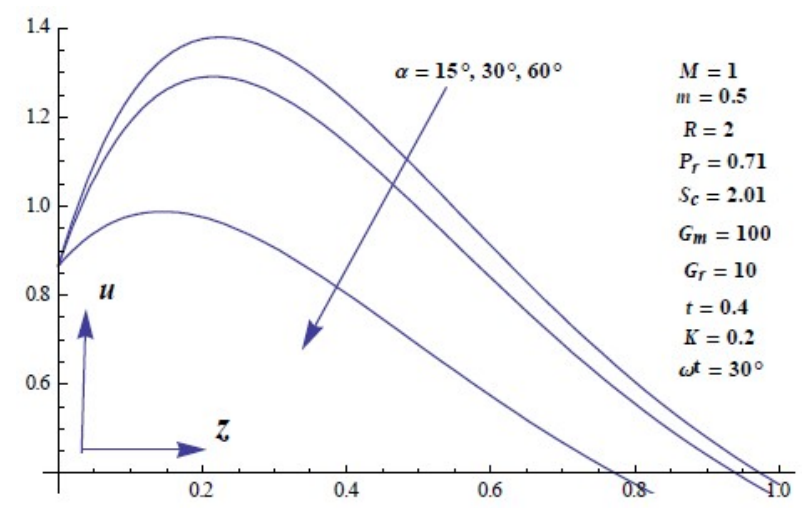

Figure 1.1. Velocity u for different values of $\alpha$.

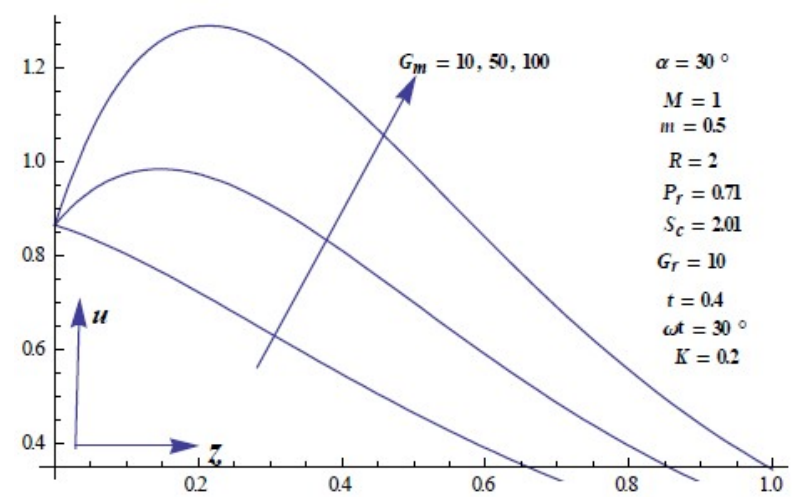

Figure 1.2. Velocity u for different values of $\mathrm{Gm}$.

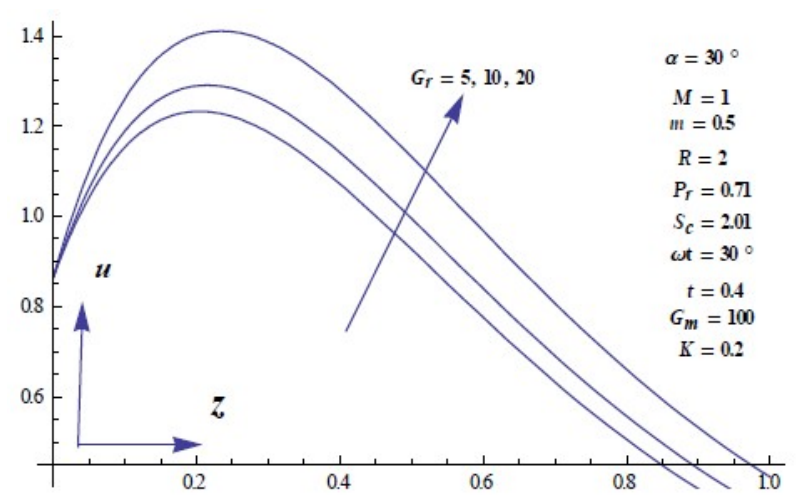

Figure 1.3. Velocity u for different values of $G r$.

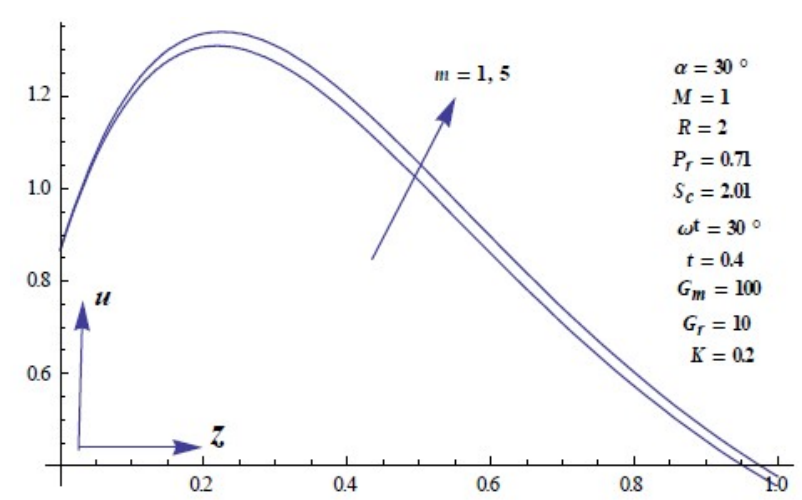

Figure 1.4. Velocity u for different values of $m$.

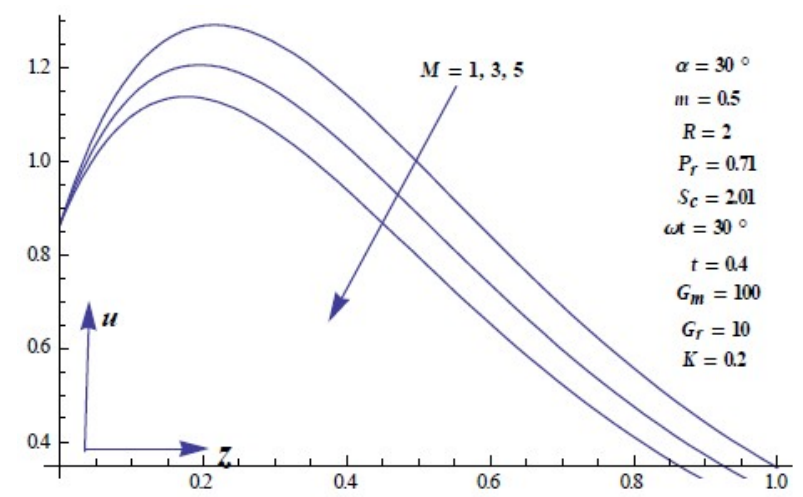

Figure 1.5. Velocity u for different values of $M$. 


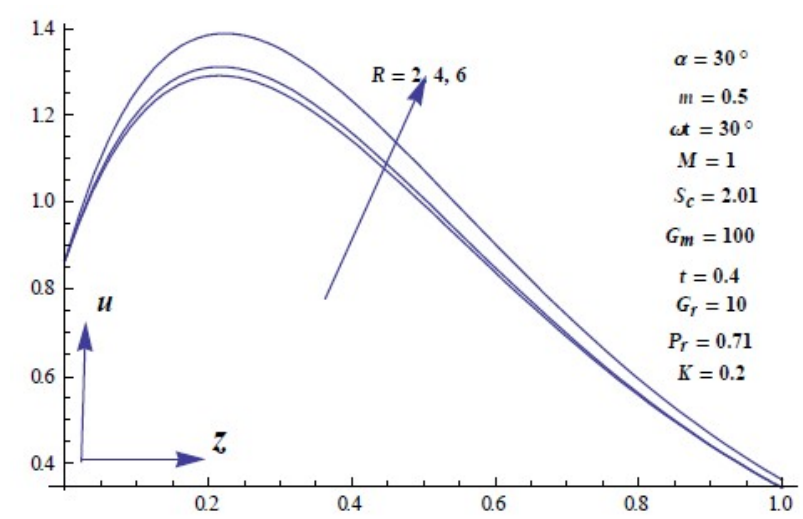

Figure 1.6 Velocity u for different values of $R$

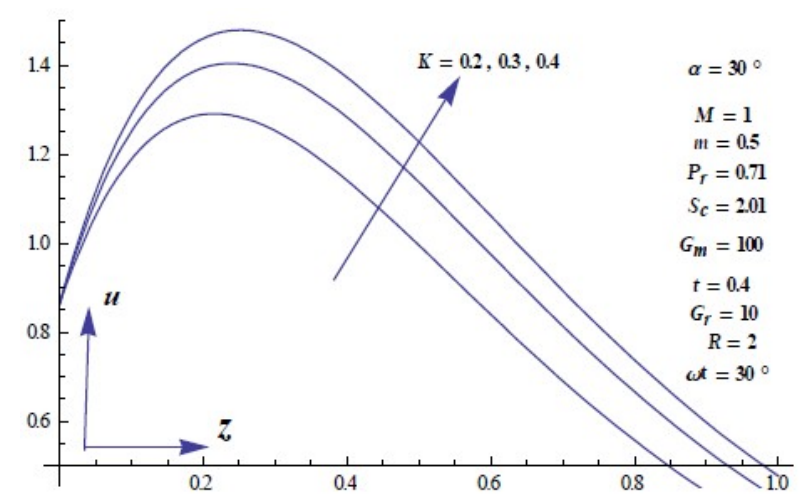

Figure 1.7. Velocity u for different values of $K$.

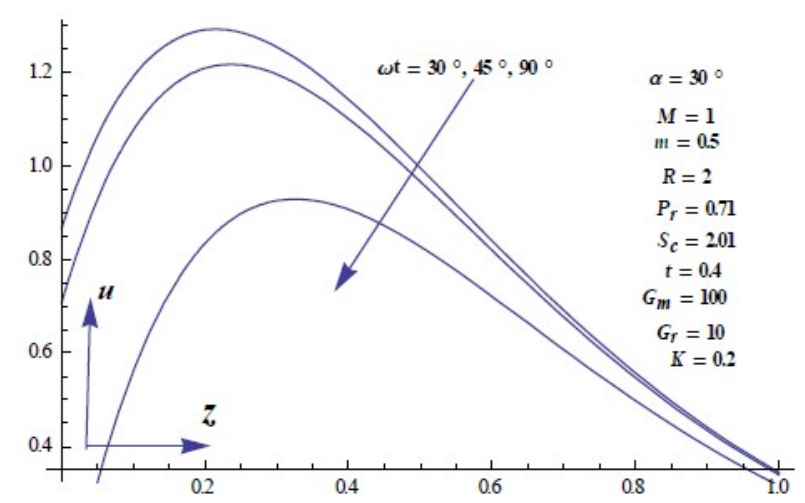

Figure 1.8. Velocity u for different values of $\omega t$.

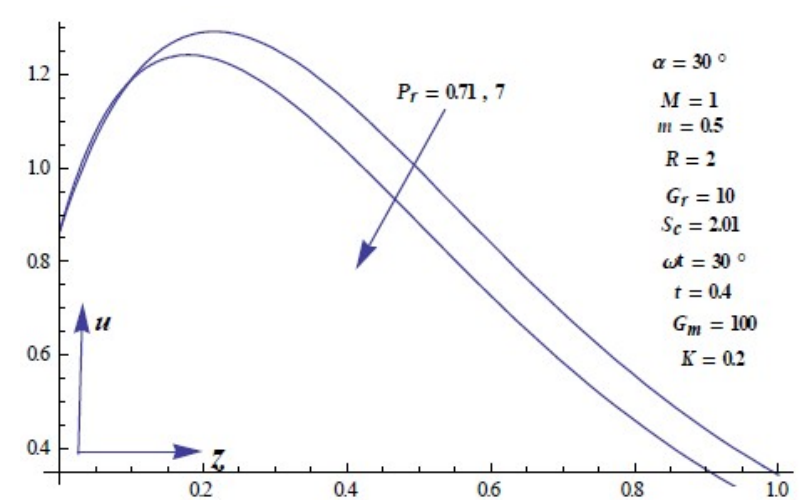

Figure 1.9. Velocity u for different values of $P r$.

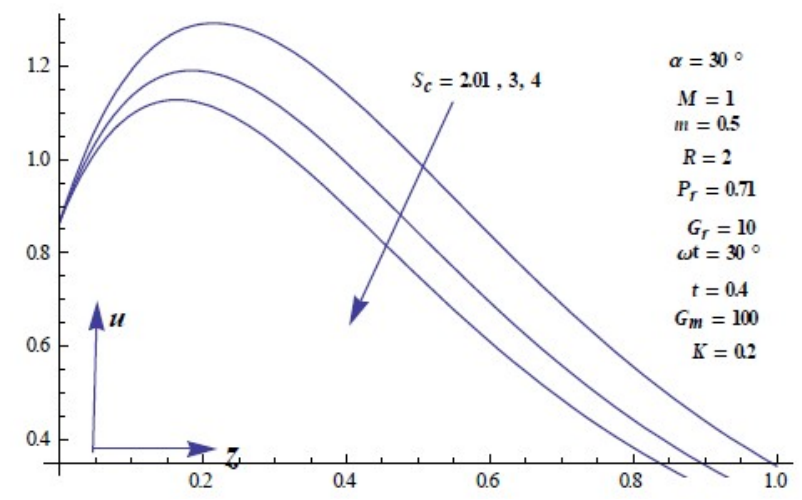

Figure 1.10. Velocity u for different values of Sc.

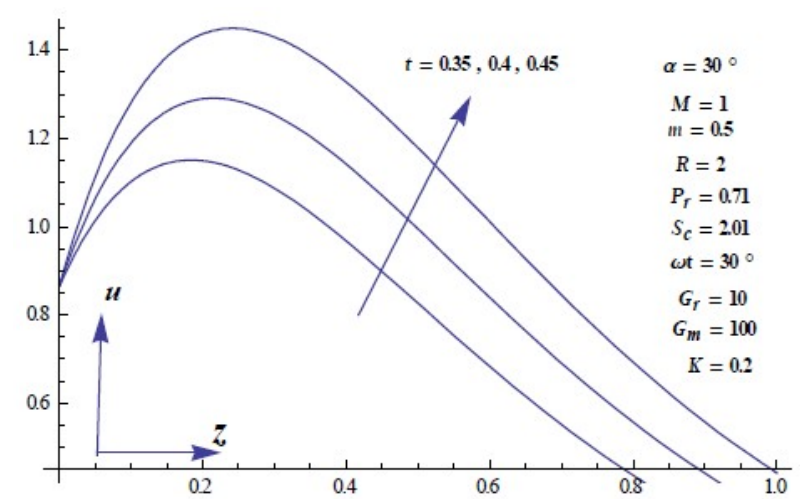

Figure 1.11. Velocity $u$ for different values of $t$.

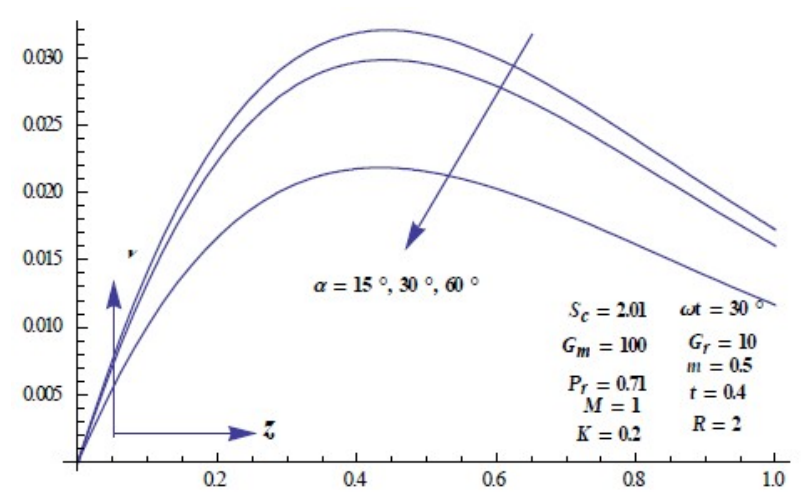

Figure 2.1. Velocity $\mathrm{v}$ for different values of $\alpha$.

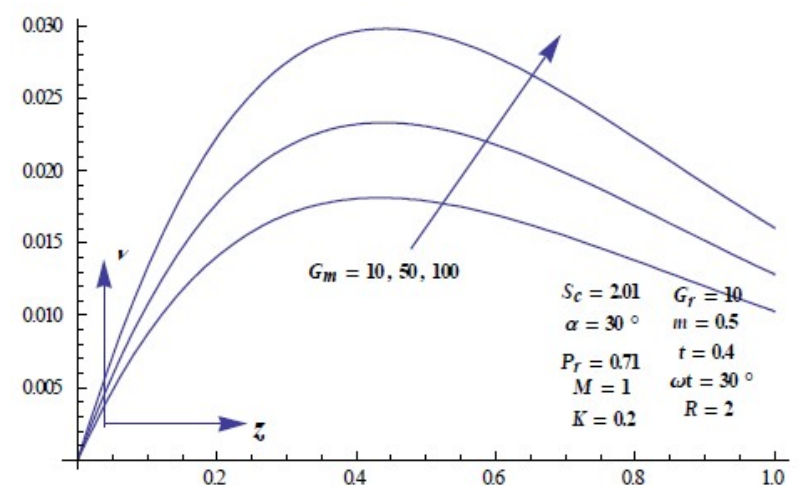

Figure 2.2. Velocity v for different values of $\mathrm{Gm}$. 


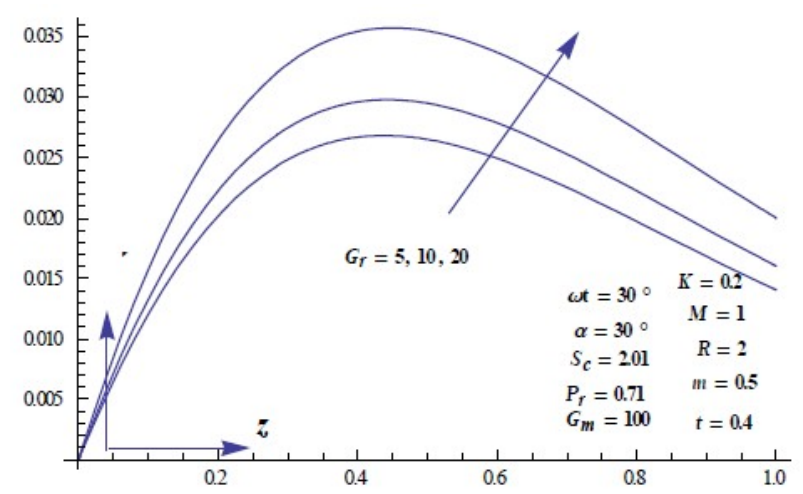

Figure 2.3. Velocity v for different values of $G r$.

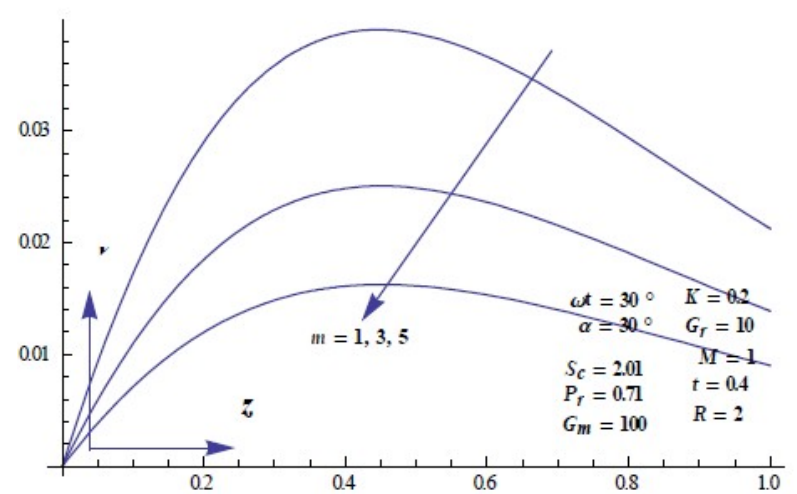

Figure 2.4. Velocity $v$ for different values of $m$.

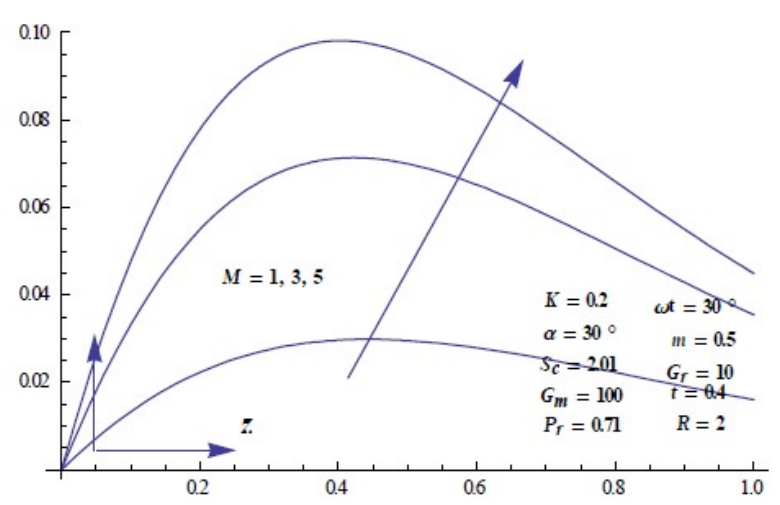

Figure 2.5. Velocity $v$ for different values of $M$.

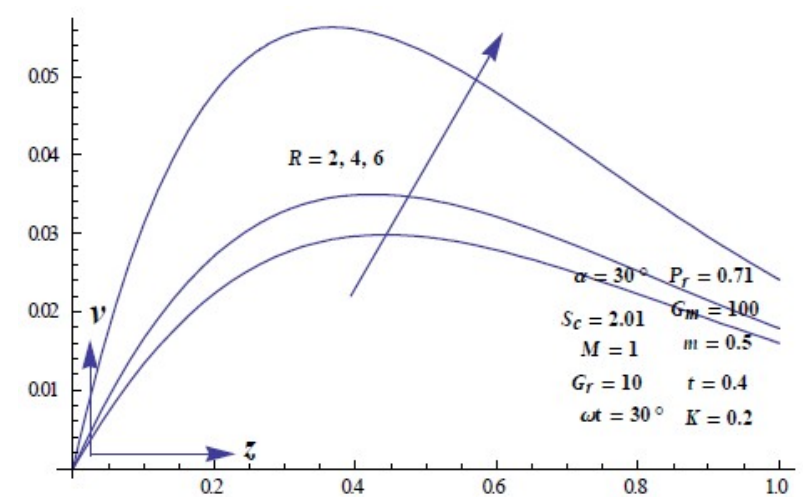

Figure 2.6. Velocity $\mathrm{v}$ for different values of $R$.

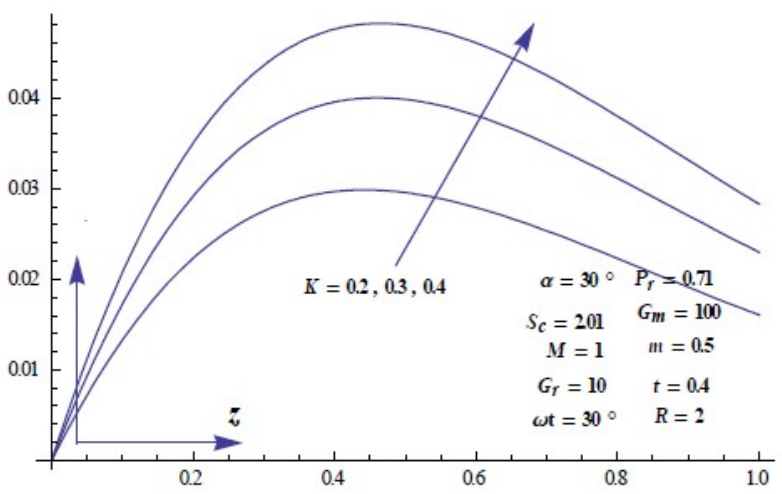

Figure 2.7. Velocity $v$ for different values of $K$.

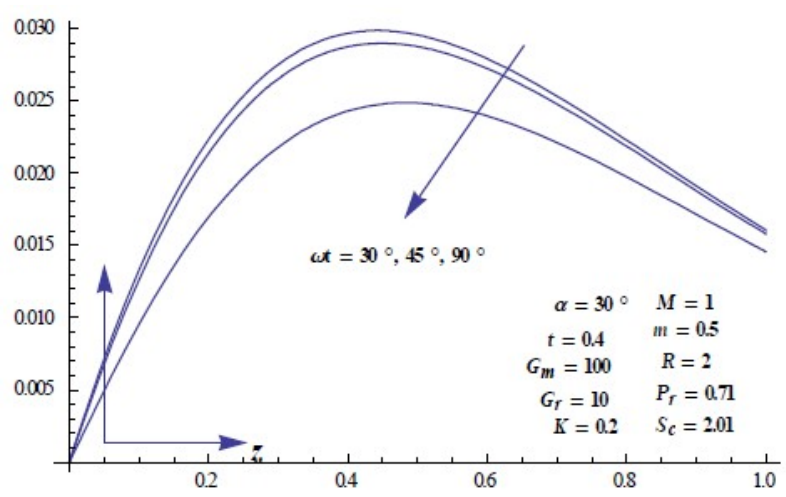

Figure 2.8. Velocity $v$ for different values of $\omega t$.

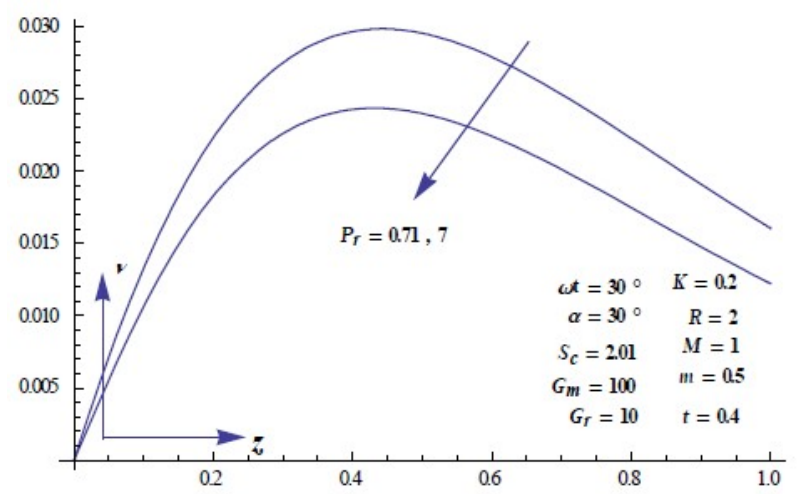

Figure 2.9. Velocity $v$ for different values of $\operatorname{Pr}$.

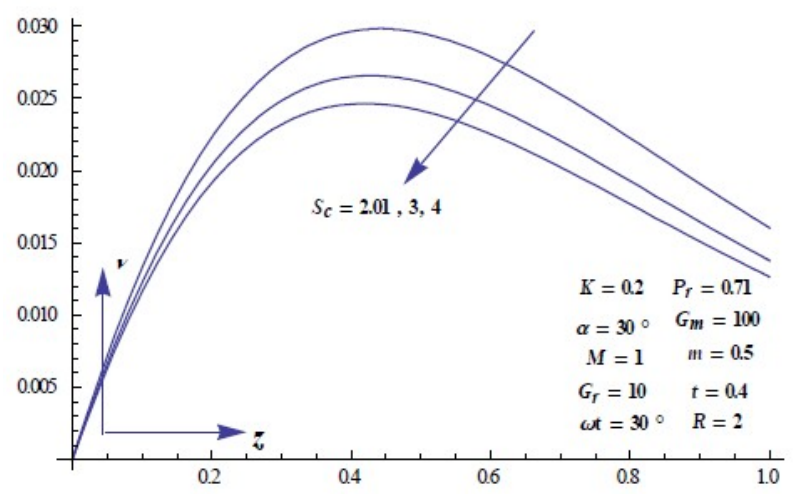

Figure 2.10. Velocity v for different values of Sc. 


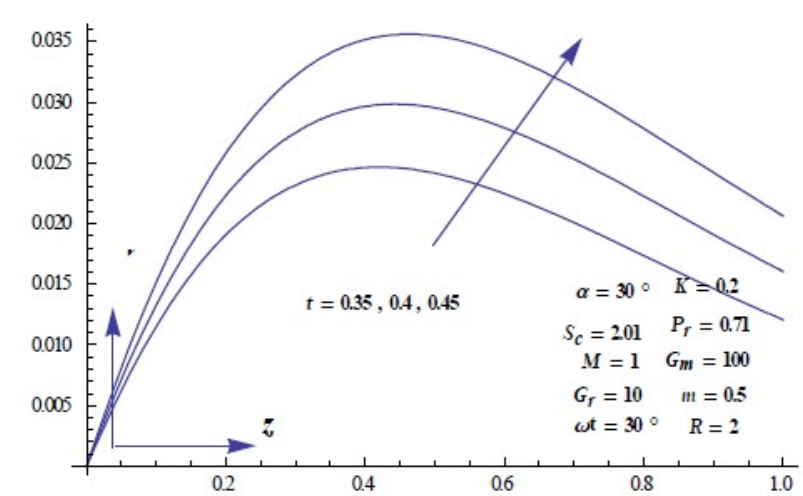

Figure 2.11. Velocity $v$ for different values of $t$.

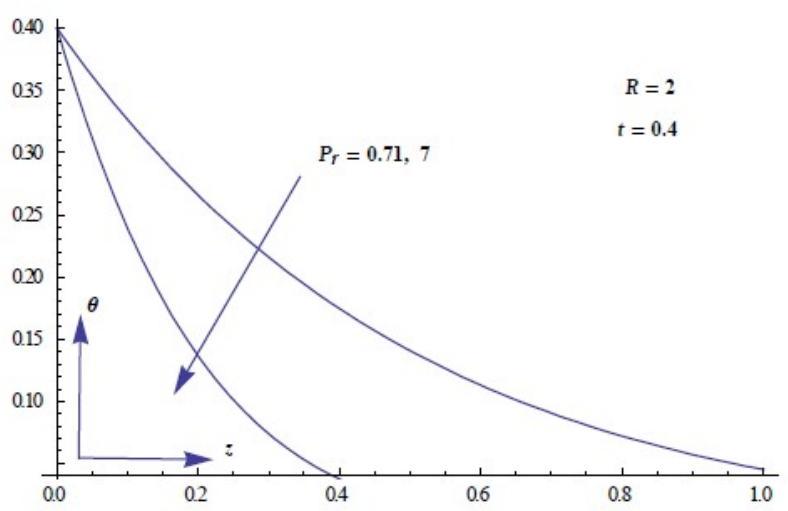

Figure 3.1. Temperature $\theta$ for different values of $\mathrm{Pr}$

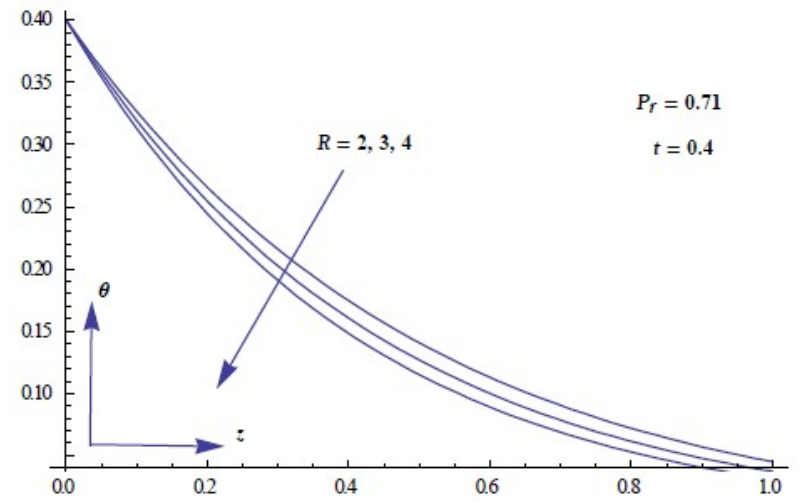

Figure 3.2. Temperature $\theta$ for different values of $R$.

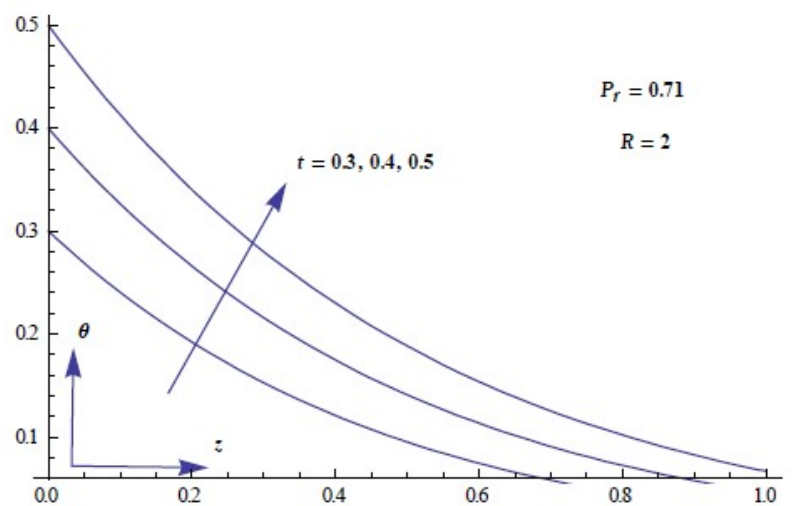

Figure 3.3. Temperature $\theta$ for different values of $t$.

Table 1 Skin friction for different parameters

\begin{tabular}{|c|c|c|c|c|c|c|c|c|c|c|c|c|}
\hline $\begin{array}{c}\alpha \\
\text { (in degrees) }\end{array}$ & $M$ & $m$ & $P r$ & $S c$ & $G m$ & $G r$ & $R$ & $\begin{array}{c}\omega t \\
\text { (in degrees) }\end{array}$ & $K$ & $t$ & $\tau_{x}$ & $\tau_{y}$ \\
\hline 15 & 2 & 1 & 0.71 & 2.01 & 100 & 010 & 2 & 30 & 0.2 & 0.2 & 3132.35 & 457.738 \\
\hline 30 & 2 & 1 & 0.71 & 2.01 & 100 & 010 & 2 & 30 & 0.2 & 0.2 & 2808.19 & 410.413 \\
\hline 60 & 2 & 1 & 0.71 & 2.01 & 100 & 010 & 2 & 30 & 0.2 & 0.2 & 1620.47 & 237.021 \\
\hline 30 & 3 & 1 & 0.71 & 2.01 & 100 & 010 & 2 & 30 & 0.2 & 0.2 & 2566.26 & 535.114 \\
\hline 30 & 5 & 1 & 0.71 & 2.01 & 100 & 010 & 2 & 30 & 0.2 & 0.2 & 2144.67 & 676.703 \\
\hline 30 & 2 & 3 & 0.71 & 2.01 & 100 & 010 & 2 & 30 & 0.2 & 0.2 & 3205.77 & 306.419 \\
\hline 30 & 2 & 5 & 0.71 & 2.01 & 100 & 010 & 2 & 30 & 0.2 & 0.2 & 3284.28 & 203.796 \\
\hline 30 & 2 & 1 & 7.00 & 2.01 & 100 & 010 & 2 & 30 & 0.2 & 0.2 & -301.104 & 3.61861 \\
\hline 30 & 2 & 1 & 0.71 & 3.00 & 100 & 010 & 2 & 30 & 0.2 & 0.2 & 2807.95 & 410.407 \\
\hline 30 & 2 & 1 & 0.71 & 2.01 & 010 & 010 & 2 & 30 & 0.2 & 0.2 & 2806.20 & 410.387 \\
\hline 30 & 2 & 1 & 0.71 & 2.01 & 050 & 010 & 2 & 30 & 0.2 & 0.2 & 2807.08 & 410.398 \\
\hline 30 & 2 & 1 & 0.71 & 2.01 & 100 & 050 & 2 & 30 & 0.2 & 0.2 & 14040.1 & 2051.29 \\
\hline 30 & 2 & 1 & 0.71 & 2.01 & 100 & 100 & 2 & 30 & 0.2 & 0.2 & 28079.9 & 4102.39 \\
\hline 30 & 2 & 1 & 0.71 & 2.01 & 100 & 010 & 4 & 30 & 0.2 & 0.2 & 3856.76 & 709.173 \\
\hline 30 & 2 & 1 & 0.71 & 2.01 & 100 & 010 & 6 & 30 & 0.2 & 0.2 & 5740.37 & 1341.01 \\
\hline 30 & 2 & 1 & 0.71 & 2.01 & 100 & 010 & 2 & 45 & 0.2 & 0.2 & 2808.82 & 410.395 \\
\hline 30 & 2 & 1 & 0.71 & 2.01 & 100 & 010 & 2 & 60 & 0.2 & 0.2 & 2809.64 & 410.370 \\
\hline 30 & 2 & 1 & 0.71 & 2.01 & 100 & 010 & 2 & 30 & 0.5 & 0.2 & 4652.68 & 963.903 \\
\hline 30 & 2 & 1 & 0.71 & 2.01 & 100 & 010 & 2 & 30 & 1.0 & 0.2 & 5741.23 & 1341.05 \\
\hline 30 & 2 & 1 & 0.71 & 2.01 & 100 & 010 & 2 & 30 & 0.2 & 0.3 & 4813.21 & 856.901 \\
\hline 30 & 2 & 1 & 0.71 & 2.01 & 100 & 010 & 2 & 30 & 0.2 & 0.4 & 2809.64 & 410.370 \\
\hline
\end{tabular}

Table 2Nusselt number for different parameters.

\begin{tabular}{|c|c|c|c|}
\hline$P r$ & $R$ & $t$ & $N u$ \\
\hline 0.71 & 2 & 0.4 & -0.805273 \\
\hline 7.00 & 2 & 0.4 & -1.959260 \\
\hline 0.71 & 3 & 0.4 & -0.894014 \\
\hline 0.71 & 4 & 0.4 & -0.976083 \\
\hline 0.71 & 2 & 0.5 & -0.950956 \\
\hline 0.71 & 2 & 0.6 & -1.094940 \\
\hline
\end{tabular}




\section{CONCLUSION}

The conclusions of the study are as follows:

- Primary velocity increases with the increase in $G r, G m, m, R, K$ and $t$.

- $\quad$ Primary velocity decreases with $\alpha, M, \omega t, P r$ and $S c$.

- Secondary velocity increases with the increase in $G r, G m, M, R$, $K$ and $t$.

- $\quad$ Secondary velocity decreases with $\alpha, m, \omega t, P r$ and $S c$.

- $\boldsymbol{\tau}_{x}$ increases with the increase in $G r, G m, m, \omega t, K$ and $R$, and it decreases with $\alpha, M, P r, S c$ and $t$.

- $\boldsymbol{\tau}_{y}$ increases with the increase in $G r, G m, R, M$ and $K$, and it decreases with $\alpha, m, \omega t, P r, S c$ and $t$.

- $\quad N u$ decreases with increase in $P r, R$ and $t$.

\section{REFERENCES}

[1] D. Pal and H. Mondal, Meccanica 44: (2009), 133-144.

[2] K. Manivannan, R. Muthucumarswamy and V. Thangaraj,Thermalscience, Vol.13, No.2, (2009), 155-162.

[3] P. Vyas and N. Srivastava, Applied Mathematical Sciences, Vol. 4, No.50, (2010), 2475-2484.

[4] U.S. Rajput and S. Kumar, International Journal of Mathematical Archive-4(10): (2013), 106-114.

[5] S. Purkayastha and R. Choudhury, WSEAS Transactions on Applied and Theoretical Mechanics, Volume 9, (2014), 196-205.

[6] Z. Ismail, I. Khan, A. Imran, A. Hussanan and H. Shafie, Malaysian Journal of Fundamental and Applied Sciences Vol.10, No.1 (2014) 3742.

[7] M. Srinivas and N. Kishan, World Applied Sciences Journal 33 (6): (2015), 1032-104.

[8] N. Ananda Reddy, M.C. Raju, S.V.K. Varma, (Elsevier) Ain Shams Engineering Journal (2014) 5, 1361-1369.

[9] R.Swetha, G.Viswanatha Reddy, S.Vijaya Kumar Varma, (Elsevier) Procedia Engineering 127 (2015) 553 - 560.

[10] Chandra Shekar Balla, Kishan Naikoti, (Elsevier) Alexandria Engineering Journal (2015) 54, 661-671. 


\section{APPENDIX}

$A_{1}=1+e^{2 \sqrt{a} z}\left(1-A_{18}\right)-A_{17}, A_{2}=-A_{1}, A_{3}=1-e^{2 \sqrt{a} z}\left(1-A_{18}\right)-A_{17}, A_{4}=-1+A_{19}+A_{30}\left(A_{20}-1\right)$, $A_{5}=-1+A_{21}+A_{28}\left(A_{22}-1\right), A_{6}=-1+A_{23}+A_{26}\left(A_{31}-1\right), A_{7}=-1+A_{29}+A_{27}\left(A_{30}-1\right), A_{8}=-1+A_{23}+A_{26}\left(A_{31}-1\right)$, $A_{9}=-1-A_{24}-A_{28}\left(1-A_{25}\right), A_{10}=-A_{9}$,

$A_{12}=\exp \left(\frac{a t}{P_{r}-1}-\frac{R t}{P_{r}-1}-z \sqrt{\frac{a P_{r}-R}{P_{r}-1}}\right)$

$A_{13}=\exp \left(\frac{a t}{S_{c}-1}-z \sqrt{\frac{a S_{c}}{S_{c}-1}}\right), A_{12}=\exp \left(\frac{a t}{P_{r}-1}-\frac{R t}{P_{r}-1}-A b s[z] \sqrt{\frac{P_{r}\left(a P_{r}-R\right)}{P_{r}-1}}\right), A_{15}=-1+\operatorname{erf}\left[\frac{z \sqrt{S_{c}}}{2 \sqrt{t}}\right]$,

$A_{16}=\exp \left(A b s[z] \sqrt{P_{r} R}\right), A_{17}=\operatorname{erf}\left[\frac{2 \sqrt{a} t-z}{2 \sqrt{t}}\right], A_{18}=\operatorname{erf}\left[\frac{2 \sqrt{a} t+z}{2 \sqrt{t}}\right], A_{19}=\operatorname{erf}\left[\frac{z-2 t \sqrt{\frac{a P_{r}-R}{P_{r}-1}}}{2 t}\right]$,

$A_{20}=\operatorname{erf}\left[\frac{z+2 t \sqrt{\frac{a P_{r}-R}{P_{r}-1}}}{2 t}\right]$,

$A_{21}=\operatorname{erf}\left[\frac{z-2 t \sqrt{\frac{a S_{c}}{S_{c}-1}}}{2 t}\right], A_{22}=\operatorname{erf}\left[\frac{z+2 t \sqrt{\frac{a S_{c}}{S_{c}-1}}}{2 t}\right], A_{23}=\operatorname{erf}\left[\frac{\operatorname{Abs}[z] \cdot A b s\left[P_{r}\right]}{2 \sqrt{t}}-\sqrt{\frac{t R}{P_{r}}}\right], A_{24}=\operatorname{erf}\left[\frac{2 t \sqrt{\frac{a}{S_{c}-1}}-2 \sqrt{S_{c}}}{2 t}\right]$,

$A_{25}=\operatorname{erf}\left[\frac{2 t \sqrt{\frac{a}{S_{c}-1}}+2 \sqrt{S_{c}}}{2 t}\right], A_{16}=\exp \left(A b s[z] \sqrt{P_{r} R}\right), A_{27}=\exp \left(2 A b s[z] \sqrt{\frac{P_{r}\left(a P_{r}-R\right)}{P_{r}-1}}\right), A_{28}=e^{-2 z \sqrt{\frac{a S_{c}}{S_{c}-1}}}$,

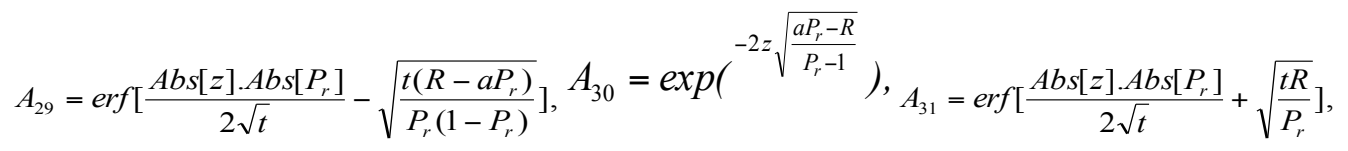

$A_{32}=\operatorname{erf}\left[\frac{A b s[z] \cdot A b s\left[P_{r}\right]}{2 \sqrt{t}}+\sqrt{\frac{t\left(R-a P_{r}\right)}{P_{r}\left(1-P_{r}\right)}}\right], \begin{aligned} & A_{33}=A_{34}+A_{35}-\exp (-z \sqrt{a+i \omega}) A_{36} \\ & -\exp (-z \sqrt{a+i \omega}+2 i t \omega) A_{37},\end{aligned}$

$\begin{aligned} A_{34}=\exp (-z \sqrt{a+i \omega})+\exp (-z \sqrt{a-i \omega}), & \begin{array}{l}A_{35}=\exp (-z \sqrt{a+i \omega}+2 i t \omega) \\ +\exp (z \sqrt{a-i \omega}+2 i t \omega),\end{array}\end{aligned}$

$A_{36}=\operatorname{erf}\left[\frac{z-2 t \sqrt{a-i \omega}}{2 \sqrt{t}}\right]+\operatorname{erf}\left[\frac{z+2 t \sqrt{a-i \omega}}{2 \sqrt{t}}\right], A_{37}=\operatorname{erf}\left[\frac{z-2 t \sqrt{a+i \omega}}{2 \sqrt{t}}\right]+\operatorname{erf}\left[\frac{z+2 t \sqrt{a+i \omega}}{2 \sqrt{t}}\right]$ 